\title{
Author Correction: Tyrosine dephosphorylated cortactin downregulates contractility at the epithelial zonula adherens through SRGAP1
}

\author{
Xuan Liang${ }^{1}$, Srikanth Budnar', Shafali Gupta1, Suzie Verma', Siew-Ping Han', Michelle M. Hill², Roger J. Daly ${ }^{3}$, \\ Robert G. Parton (10) ${ }^{1,4,5}$, Nicholas A. Hamilton ${ }^{1}$, Guillermo A. Gomez ${ }^{1,6}$ \& Alpha S. Yap ${ }^{1,4}$
}

Nature Communications 8:790 10.1038/s41467-017-00797-w; Article published online: 5 October 2017

Previous work by Garcia Ponce et al. and Citalán-Madrid et al. suggesting a link between cortactin and RhoA-mediated actomyosin contractility was inadvertently omitted from the original version of this manuscript and should have been cited within the "Discussion" section as follows:

"Previous studies in cortactin-null endothelium and intestinal epithelium revealed hyperactivated RhoA-mediated contractility in these cell types, contrasting with the decrease in junctional RhoA signaling and contractility that we observed, but the direct involvement of cortactin in mediating actomyosin contractility was not established ${ }^{1,2}$. Future research will have to explore the compatibility of these observations."

1. Garcia Ponce, A. et al. Loss of cortactin causes endothelial barrier dysfunction via disturbed adrenomedullin secretion and actomyosin contractility. Sci Rep. 6, 29003 (2016).

2. Citalán-Madrid, A. F. et al. Cortactin deficiency causes increased RhoA/ROCK1-dependent actomyosin contractility, intestinal epithelial barrier dysfunction, and disproportionately severe DSS-induced colitis. Mucosal Immunol. 10, 1237-1247 (2017).

Published online: 05 December 2017

\begin{abstract}
cc reproduction in any medium or format, as long as you give appropriate credit to the original author(s) and the source, provide a link to the Creative Commons license, and indicate if changes were made. The images or other third party material in this article are included in the article's Creative Commons license, unless indicated otherwise in a credit line to the material. If material is not included in the article's Creative Commons license and your intended use is not permitted by statutory regulation or exceeds the permitted use, you will need to obtain permission directly from the copyright holder. To view a copy of this license, visit http://creativecommons.org/licenses/by/4.0/.
\end{abstract}

(c) The Author(s) 2017

\footnotetext{
${ }^{1}$ Division of Cell Biology and Molecular Medicine, The University of Queensland, St. Lucia, QLD 4072, Australia. ${ }^{2}$ The University of Queensland Diamantina Institute, Brisbane, QLD 4102, Australia. ${ }^{3}$ Cancer Program, Biomedicine Discovery Institute and Department of Biochemistry and Molecular Biology, Monash University, Clayton, VIC 3800, Australia. ${ }^{4}$ Program in Membrane Interface Biology, Institute for Molecular Bioscience, The University of Queensland, St. Lucia, QLD 4072, Australia. ${ }^{5}$ Centre for Microscopy and Microanalysis, The University of Queensland, St. Lucia, QLD 4072, Australia. ${ }^{6}$ Present address: Centre for Cancer Biology, SA Pathology and the University of South Australia, Adelaide, SA 5000, Australia Correspondence and requests for materials should be addressed to G.A.G. (email: guillermo.gomez@unisa.edu.au) or to A.S.Y. (email: a.yap@uq.edu.au)
} 